\title{
ON THE GLOBAL ASYMPTOTIC STABILITY OF A TWO DIMENSIONAL SYSTEM OF DIFFERENCE EQUATIONS WITH QUADRATIC TERMS
}

\author{
ERKAN TAŞDEMIR
}

\begin{abstract}
In this paper, we study the global asymptotically stability of following system of difference equations with quadratic terms:

$$
x_{n+1}=A+B \frac{y_{n}}{y_{n-1}^{2}}, y_{n+1}=A+B \frac{x_{n}}{x_{n-1}^{2}}
$$

where $A$ and $B$ are positive numbers and the initial values are positive numbers. We also investigate the rate of convergence and oscillation behaviour of the solutions of related system.
\end{abstract}

\section{INTRODUCTION}

Over the last two decades, difference equations or their systems have been huge attention among researchers which is mathematician or not. Moreover difference equations or systems have too many applications between many branches of science. For example, in [13] Khan et al studied global dynamics of an one-dimensional discrete-time laser model. Further in [7] Din et al studied stability of a discrete ecological model. There are many examples related to applications of difference equations or systems. Therefore, studies on difference equations are increasing day by day and will continue to increase. Additionally, there are many papers related to our study as follows:

In [24], Yang et al investigated the solutions, stability and asymptotic behavior of the system of the two nonlinear difference equations

$$
x_{n+1}=\frac{A x_{n}}{1+y_{n}^{p}}, y_{n+1}=\frac{B y_{n}}{1+x_{n}^{p}} .
$$

In [8], Elabbasy et al studied the global behaviour of following system of difference equations

$$
x_{n+1}=\frac{a_{1} x_{n}}{a_{2}+a_{3} y_{n}^{r}}, y_{n+1}=\frac{b_{1} y_{n}}{b_{2}+b_{3} x_{n}^{r}} .
$$

In [3], Bacani et al considered solutions of the following two nonlinear difference equations

$$
x_{n+1}=\frac{q}{p+x_{n}^{v}}, y_{n+1}=\frac{q}{-p+y_{n}^{v}} .
$$

1991 Mathematics Subject Classification. 39A10, 39A23, 39A30.

Key words and phrases. Difference equations, dynamical systems, global stability, rate of convergence, boundedness, oscillation. 
In [11], Hadziabdic et al studied global behaviours of following system of difference equations

$$
x_{n+1}=\frac{b_{1} x_{n}^{2}}{A_{1}+y_{n}^{2}}, y_{n+1}=\frac{a_{2}+c_{2} y_{n}^{2}}{x_{n}^{2}} .
$$

In [5], Burgic et al investigated the global stability properties and asymptotic behavior of solutions for the system of difference equations

$$
x_{n+1}=\frac{x_{n}}{a+y_{n}^{2}}, y_{n+1}=\frac{y_{n}}{b+x_{n}^{2}} .
$$

In [4], Beso et al studied boundedness of solutions of following difference equation

$$
x_{n+1}=\gamma+\delta \frac{x_{n}}{x_{n-1}^{2}} .
$$

They also investigated global asymptotic stability of relatd difference equation.

Motivated by difference equations and their systems, we consider the following system of difference equations

$$
x_{n+1}=A+B \frac{y_{n}}{y_{n-1}^{2}}, y_{n+1}=A+B \frac{x_{n}}{x_{n-1}^{2}}
$$

where $A$ and $B$ are positive numbers and the initial values are positive numbers. In this paper we study the stability, global behaviour and rate of convergence of solutions of system (1.1). We also investigate the oscillation behaviour of solutions of related system.

In this here, we give two theorems which are used during this study.

Theorem 1.1 (Linearized Stability Theorem [16], p.11). Assume that

$$
X_{n+1}=F\left(X_{n}\right), n=0,1, \cdots
$$

is a system of difference equations such that $\bar{X}$ is a fixed point of $F$.

(i): If all eigenvalues of the Jacobian matrix $B$ about $\bar{X}$ lie inside the open unit disk $|\lambda|<1$, that is, if all of them have absolute value less than one, then $\bar{X}$ is locally asymptotically stable.

(ii): If at least one of them has a modulus greater than one, then $\bar{X}$ is unstable.

Theorem 1.2. [2] Let $n \in N_{n_{0}}^{+}$and $g(n, u, v)$ be a decreasing function in $u$ and $v$ for any fixed $n$. Suppose that for $n \geq n_{0}$, the inqualities

$$
\begin{aligned}
& y_{n+1} \leq g\left(n, y_{n}, y_{n-1}\right) \\
& u_{n+1} \geq g\left(n, y_{n}, y_{n-1}\right)
\end{aligned}
$$

hold. Then

$$
y_{n_{0}-1} \leq u_{n_{0}-1}, y_{n_{0}} \leq u_{n_{0}}
$$

implies that

$$
y_{n} \leq u_{n}, n \geq n_{0} .
$$




\section{Linearized Stability of System}

First of all, we consider the change of the variables for system (1.1) as follows:

$$
t_{n}=\frac{x_{n}}{A}, z_{n}=\frac{y_{n}}{A} .
$$

From this, system (1.1) transform into following system:

$$
t_{n+1}=1+p \frac{z_{n}}{z_{n-1}^{2}}, z_{n+1}=1+p \frac{t_{n}}{t_{n-1}^{2}}
$$

where $p=\frac{B}{A^{2}}>0$. From now on, we study the system (2.1).

Lemma 2.1. Let $p>0$. Unique positive equilibrium point of system (2.1) is

$$
(\bar{t}, \bar{z})=\left(\frac{1+\sqrt{1+4 p}}{2}, \frac{1+\sqrt{1+4 p}}{2}\right) .
$$

Now, we consider a transformation as follows:

$$
\left(t_{n}, t_{n-1}, z_{n}, z_{n-1}\right) \rightarrow\left(f, f_{1}, g, g_{1}\right)
$$

where $f=1+p \frac{z_{n}}{z_{n-1}^{2}}, f_{1}=t_{n}, g=1+p \frac{t_{n}}{t_{n-1}^{2}}, g_{1}=z_{n}$. Thus we get the jacobian matrix about equilibrium point $(\bar{t}, \bar{z})$ :

$$
B(\bar{t}, \bar{z})=\left(\begin{array}{cccc}
0 & 0 & \frac{p}{\bar{z}^{2}} & \frac{-2 p}{\bar{z}^{2}} \\
1 & 0 & 0 & 0 \\
\frac{p}{t^{2}} & \frac{-2 p}{t^{2}} & 0 & 0 \\
0 & 0 & 1 & 0
\end{array}\right) .
$$

Thus, the linearized system of system (2.1) about the unique positive equilibrium point is given by $X_{N+1}=B(\bar{t}, \bar{z}) X_{N}$, where

$$
\begin{aligned}
X_{N} & =\left(\begin{array}{c}
t_{n} \\
t_{n-1} \\
z_{n} \\
z_{n-1}
\end{array}\right), \\
B(\bar{t}, \bar{z}) & =\left(\begin{array}{cccc}
0 & 0 & \frac{p}{\bar{z}^{2}} & \frac{-2 p}{\bar{z}^{2}} \\
1 & 0 & 0 & 0 \\
\frac{p}{t^{2}} & \frac{-2 p}{t^{2}} & 0 & 0 \\
0 & 0 & 1 & 0
\end{array}\right) .
\end{aligned}
$$

Hence, the characteristic equation of $B(\bar{t}, \bar{z})$ about the unique positive equilibrium point $(\bar{t}, \bar{z})$ is

$$
\lambda^{4}-\frac{p^{2}}{\bar{t}^{2} \bar{z}^{2}} \lambda^{2}+\frac{4 p^{2}}{\bar{t}^{2} \bar{z}^{2}} \lambda-\frac{4 p^{2}}{\bar{t}^{2} \bar{z}^{2}}=0
$$

Due to $\bar{t}=\bar{z}$, we can rearrange the characteristic equation such that

$$
\lambda^{4}-\frac{p^{2}}{\bar{t}^{4}} \lambda^{2}+\frac{4 p^{2}}{\bar{t}^{4}} \lambda-\frac{4 p^{2}}{\bar{t}^{4}}=0 .
$$


Therefore, we obtain the four roots of characteristic equation as follows:

$$
\begin{aligned}
& \lambda_{1}=\frac{p+\sqrt{p^{2}-8 p \bar{t}^{2}}}{2 \bar{t}^{2}}, \\
& \lambda_{2}=\frac{p-\sqrt{p^{2}-8 p \bar{t}^{2}}}{2 \bar{t}^{2}}, \\
& \lambda_{3}=\frac{-p+\sqrt{p^{2}+8 p \bar{t}^{2}}}{2 \bar{t}^{2}}, \\
& \lambda_{4}=\frac{-p-\sqrt{p^{2}+8 p \bar{t}^{2}}}{2 \bar{t}^{2}} .
\end{aligned}
$$

Now, we calculate $\bar{t}^{2}$ and write in $\lambda_{1}$. Then we have

$$
\begin{aligned}
\lambda_{1} & =\frac{p+\sqrt{p^{2}-4 p(1+2 p+\sqrt{4 p+1})}}{1+2 p+\sqrt{4 p+1}} \\
& =\frac{p+\sqrt{-7 p^{2}-4 p-4 p \sqrt{1+4 p}}}{1+2 p+\sqrt{4 p+1}} \\
& =\frac{p+\sqrt{7 p^{2}+4 p+4 p \sqrt{1+4 p}} i}{1+2 p+\sqrt{4 p+1}} .
\end{aligned}
$$

Thus straightforward calculations show that

$$
\left|\lambda_{1}\right|=\frac{2 \sqrt{2 p}}{1+\sqrt{1+4 p}} .
$$

Additionally, we obtain similarly calculations that

$$
\left|\lambda_{2}\right|=\frac{2 \sqrt{2 p}}{1+\sqrt{1+4 p}} .
$$

On the other hand, we consider $\lambda_{3}$ as follows:

$$
\begin{aligned}
\lambda_{3} & =\frac{-p+\sqrt{9 p^{2}+4 p+4 p \sqrt{4 p+1}}}{1+2 p+\sqrt{4 p+1}} \\
& =\frac{-p+\sqrt{(3 p+\sqrt{1+4 p})^{2}-1-2 p \sqrt{4 p+1}}}{1+2 p+\sqrt{4 p+1}} \\
& <\frac{-p+\sqrt{(3 p+\sqrt{1+4 p})^{2}}}{1+2 p+\sqrt{4 p+1}} \\
& =\frac{2 p+\sqrt{1+4 p}}{1+2 p+\sqrt{4 p+1}}<1 .
\end{aligned}
$$

Moreover we clearly see that $\lambda_{3}>0$. So $0<\lambda_{3}<1$ for all $p>0$. Similar calculations we have that $-1<\lambda_{4}<0$ for all $p>0$.

Theorem 2.2. Suppose that $p>0$. Then the following cases hold for system (2.1):

i.: If $p<2$ then the equilibrium point of system (2.1) is locally asymptotically stable.

ii.: If $p=2$ then the equilibrium point of system (2.1) is a non-hyperbolic equilibrium.

iii.: If $p>2$ then the equilibrium point of system (2.1) is a repeller. 
Proof. Firstly we know that $\left|\lambda_{3}\right|,\left|\lambda_{4}\right|<1$ for all $p>0$. Now we consider

$$
\left|\lambda_{1}\right|=\left|\lambda_{2}\right|=\frac{2 \sqrt{2 p}}{1+\sqrt{1+4 p}} .
$$

If the equilibrium point of system (2.1) is locally asymptotically stable, then all roots of characteristic equation must lie the unit disk. Therefore, we must show that $\left|\lambda_{1}\right|,\left|\lambda_{2}\right|<1$. Hence

$$
\left|\lambda_{1}\right|=\left|\lambda_{2}\right|=\frac{2 \sqrt{2 p}}{1+\sqrt{1+4 p}}<1 .
$$

Thus, we have $2 \sqrt{2 p}<1+\sqrt{1+4 p}$. From this, we obtain that $p<2$. The proofs of other cases can be obtained in a similar way.

\section{An oscillation Result of SOlutions of System (2.1)}

In this here, we investigate the oscillation behaviour of solutions of system(2.1).

Theorem 3.1. Let $\left\{\left(t_{n}, z_{n}\right)\right\}$ be a positive solution of system (2.1) and $p>0$. Then for any $n \geq 0$ the following cases are true.

i.: If $t_{n+1}, z_{n}<\bar{t}=\bar{z}<t_{n}, z_{n+1}$ then

$$
\begin{aligned}
\left(t_{n+2 k-1}\right)_{k=1}^{\infty} & <\bar{t}<\left(t_{n+2 k}\right)_{k=1}^{\infty}, \\
\left(z_{n+2 k}\right)_{k=1}^{\infty} & <\bar{z}<\left(z_{n+2 k-1}\right)_{k=1}^{\infty} .
\end{aligned}
$$

ii.: If $t_{n}, z_{n+1}<\bar{t}=\bar{z}<t_{n+1}, z_{n}$ then

$$
\begin{aligned}
\left(t_{n+2 k}\right)_{k=1}^{\infty} & <\bar{t}<\left(t_{n+2 k-1}\right)_{k=1}^{\infty}, \\
\left(z_{n+2 k-1}\right)_{k=1}^{\infty} & <\bar{z}<\left(z_{n+2 k}\right)_{k=1}^{\infty} .
\end{aligned}
$$

Proof. Firstly we consider case (i). Assume that $t_{n+1}, z_{n}<\bar{t}=\bar{z}<t_{n}, z_{n+1}$. Then we obtain that

$$
\begin{aligned}
t_{n+2} & =1+p \frac{z_{n+1}}{z_{n}^{2}}>1+p \frac{\bar{z}}{\bar{z}^{2}}=\bar{z}=\bar{t} \\
z_{n+2} & =1+p \frac{t_{n+1}}{t_{n}^{2}}<1+p \frac{\bar{t}}{\bar{t}^{2}}=\bar{t}=\bar{z} \\
t_{n+3} & <\bar{t}, z_{n+3}>\bar{z}, t_{n+4}>\bar{t}, z_{n+4}<\bar{z}
\end{aligned}
$$

Therefore we have by using induction

$$
\begin{aligned}
t_{n}, t_{n+2}, \ldots, t_{n+2 k}, \ldots & <\bar{t}<t_{n+1}, t_{n+3}, \ldots, t_{n+2 k-1}, \ldots \\
z_{n+1}, z_{n+3}, \ldots, z_{n+2 k-1}, \ldots & <\bar{z}<z_{n}, z_{n+2}, \ldots, z_{n+2 k}, \ldots
\end{aligned}
$$

Thus the proof of (i) is completed as desired. The proof of (ii) is similar to proof of (i).

\section{Boundedness OF System (2.1)}

Lemma 4.1. Let $\left\{\left(t_{n}, z_{n}\right)\right\}$ be a positive solution of system (2.1) and $p>0$. Then $t_{n}>1$ and $z_{n}>1$ for $n \geq 1$. 
Proof. Let $\left\{\left(t_{n}, z_{n}\right)\right\}$ be a positive solution of system (2.1). Then we have from system (2.1):

$$
\begin{aligned}
& t_{1}=1+p \frac{z_{0}}{z_{-1}^{2}}>1, \\
& z_{1}=1+p \frac{t_{0}}{t_{-1}^{2}}>1 .
\end{aligned}
$$

Therefore, we obtain by induction

$$
\begin{aligned}
& t_{n+1}=1+p \frac{z_{n}}{z_{n-1}^{2}}>1, \\
& z_{n+1}=1+p \frac{t_{n}}{t_{n-1}^{2}}>1 .
\end{aligned}
$$

So, the proof of lemma is completed.

Theorem 4.2. If $0<p<1$ then every solution of system (2.1) is bounded.

Proof. Firstly we have from system (2.1) $t_{n}>1$ and $z_{n}>1$ for $n \geq 1$ and $p>0$. Moreover, every solution of system (2.1) satisfies

$$
t_{n+1} \leq 1+p+p^{2} t_{n-1}, n \geq 1,
$$

which due to Theorem 1.2 , means that $t_{n} \leq q_{n}, n=0,1, \ldots$, where $\left\{u_{n}\right\}$ satisfy

$$
u_{n+1}=1+p+p^{2} u_{n-1}, n \geq 1
$$

such that

$$
u_{s}=t_{s}, u_{s+1}=t_{s+1}, s \in\{-1,0,1, \ldots\}, n \geq s .
$$

Hence the solution $u_{n}$ of the difference equation (4.2) is

$$
u_{n}=\frac{1}{1-p}+p^{n} C_{1}+(-p)^{n} C_{2} .
$$

Moreover, we have from (4.2)

$$
u_{n+1}=1+p+p^{2} u_{n-1} \Rightarrow \lambda^{2}-p^{2}=0 \Rightarrow \lambda_{1,2}= \pm p .
$$

From this, the homogeneous solution of difference equation (4.2) is

$$
u_{h}=p^{n} C_{1}+(-p)^{n} C_{2} .
$$

In additon, from (4.2), the equilibrium solution of difference equation (4.2) is

$$
\bar{u}=1+p+p^{2} \bar{u} \Rightarrow \bar{u}=\frac{1}{1-p} .
$$

Additionally, relations (4.1) and (4.3) imply that

$$
t_{n+1}-u_{n+1} \leq p^{2}\left(t_{n-1}-u_{s-1}\right), n>s, p \in(0,1) .
$$

Therefore we have

$$
t_{n} \leq u_{n}, n>s .
$$

Hence, we obtain from (4.3), (4.4) and Lemma 4.1,

$$
1<t_{n} \leq \frac{1}{1-p}+p^{n} C_{1}+(-p)^{n} C_{2}=M_{1},
$$


where

$$
\begin{aligned}
C_{1} & =\frac{1}{2 p}\left(p t_{0}+t_{1}-\frac{1+p}{1-p}\right), \\
C_{2} & =\frac{1}{2 p}\left(p t_{0}-t_{1}+1\right) .
\end{aligned}
$$

Similarly we can write that

$$
1<z_{n} \leq \frac{1}{1-p}+p^{n} C_{3}+(-p)^{n} C_{4}=M_{2},
$$

where

$$
\begin{aligned}
C_{3} & =\frac{1}{2 p}\left(p z_{0}+z_{1}-\frac{1+p}{1-p}\right), \\
C_{4} & =\frac{1}{2 p}\left(p z_{0}-z_{1}+1\right) .
\end{aligned}
$$

\section{Convergence Results of solutions of System (2.1)}

Theorem 5.1. If $t_{n} \geq \bar{t}$ and $z_{n} \geq \bar{z}$ (resp., $t_{n} \leq \bar{t}$ and $z_{n} \leq \bar{z}$ ) for $n \geq s$ and $s \in\{-1,0, \ldots\}$ then the solution $\left\{\left(t_{n}, z_{n}\right)\right\}$ of system (2.1) tends to equilibrium point $(\bar{t}, \bar{z})$ as $n \rightarrow \infty$.

Proof. Let $\left\{\left(t_{n}, z_{n}\right)\right\}$ be a positive solution of system (2.1) such that

$$
t_{n} \geq \bar{t}, z_{n} \geq \bar{z}, n \geq s,
$$

where $s \in\{-1,0, \ldots\}$. Hence, we obtain from (5.1), system (2.1) and Lemma 4.1:

$$
t_{n+1} \leq 1+p+p^{2} t_{n-1} \text {. }
$$

Now we set

$$
u_{n+1}=1+p+p^{2} u_{n-1},
$$

such that

$$
u_{s}=t_{s}, u_{s+1}=t_{s+1}, s \in\{-1,0, \ldots\}, n \geq s .
$$

Therefore, we get from the solution of the difference equation (5.3):

$$
u_{n}=\frac{1}{1-p}+p^{n} C_{1}+(-p)^{n} C_{2}
$$

where $C_{1}, C_{2}$ depent on $t_{s}, t_{s+1}$. Moreover, we have from (5.2) and (5.3):

$$
t_{n+1}-u_{s+1} \leq p^{2}\left(t_{n-1}-u_{s-1}\right), n>s
$$

Thus we obtain from (5.4), (5.6) and by induction

$$
t_{n} \leq u_{n}, n \geq s .
$$

From (5.1), (5.5) and (5.7), we obtain that

$$
\lim _{n \rightarrow \infty} t_{n}=\bar{t}
$$

Then we similarly obtain that $\lim _{n \rightarrow \infty} z_{n}=\bar{z}$. The proof of the other case of this theorem is similar to this case, so we leave it to readers.

Theorem 5.2. Assume that $0<p<\frac{1}{2}$. Then the positive equilibrium point of system (2.1) is globally asymptotically stable. 
Proof. We have from Theorem 4.2,

$$
\begin{aligned}
& 1<l_{1}=\lim _{n \rightarrow \infty} t_{n} \leq M_{1}, \\
& 1<l_{2}=\lim _{n \rightarrow \infty} z_{n} \leq M_{2}, \\
& 1<U_{1}=\lim _{n \rightarrow \infty} t_{n} \leq M_{1}, \\
& 1<U_{2}=\lim _{n \rightarrow \infty} z_{n} \leq M_{2} .
\end{aligned}
$$

By system (2.1), we can write

$$
\begin{aligned}
U_{1} & \leq 1+p \frac{U_{2}}{l_{2}^{2}}, l_{1} \geq 1+p \frac{l_{2}}{U_{2}^{2}}, \\
U_{2} & \leq 1+p \frac{U_{1}}{l_{1}^{2}}, l_{2} \geq 1+p \frac{l_{1}}{U_{1}^{2}} .
\end{aligned}
$$

Hence we have

$$
\begin{aligned}
& U_{1}+p \frac{l_{1}}{U_{1}} \leq U_{1} l_{2} \leq l_{2}+p \frac{U_{2}}{l_{2}}, \\
& U_{2}+p \frac{l_{2}}{U_{2}} \leq U_{2} l_{1} \leq l_{1}+p \frac{U_{1}}{l_{1}} .
\end{aligned}
$$

Therefore we obtain that

$$
\begin{aligned}
U_{1}+p \frac{l_{1}}{U_{1}}+U_{2}+p \frac{l_{2}}{U_{2}} & \leq l_{2}+p \frac{U_{2}}{l_{2}}+l_{1}+p \frac{U_{1}}{l_{1}}, \\
U_{1}+p \frac{l_{1}}{U_{1}}+U_{2}+p \frac{l_{2}}{U_{2}}-l_{2}-p \frac{U_{2}}{l_{2}}-l_{1}-p \frac{U_{1}}{l_{1}} & \leq 0, \\
\left(U_{1}-l_{1}\right)\left(1-p\left(\frac{1}{l_{1}}+\frac{1}{U_{1}}\right)\right)+\left(U_{2}-l_{2}\right)\left(1-p\left(\frac{1}{l_{2}}+\frac{1}{U_{2}}\right)\right) & \leq 0 .
\end{aligned}
$$

In this here if $p \in\left(0, \frac{1}{2}\right)$ then

Thus, we get that

$$
\begin{aligned}
& 1-p\left(\frac{1}{l_{1}}+\frac{1}{U_{1}}\right)>0 \\
& 1-p\left(\frac{1}{l_{2}}+\frac{1}{U_{2}}\right)>0 .
\end{aligned}
$$

$$
U_{1}-l_{1}=0, U_{2}-l_{2}=0 .
$$

So, $U_{1}=l_{1}$ and $U_{2}=l_{2}$. The proof is completed as desired.

6. Rate of Convergence of System (2.1)

Now we study the rate of convergence of system (2.1). Hence, we consider the following system:

$$
E_{n+1}=(A+B(n)) E_{n},
$$

where $E_{n}$ is a $k$-dimensional vector, $A \in C^{k \times k}$ is a constant matrix, and $B: \mathbb{Z}^{+} \rightarrow$ $C^{k \times k}$ is a matrix function satisfying

$$
\|B(n)\| \rightarrow 0
$$

as $n \rightarrow \infty$, where $\|\cdot\|$ denotes any matrix norm that is associated with the vector norm

$$
\|(x, y)\|=\sqrt{x^{2}+y^{2}} .
$$


Theorem 6.1 (Perron's Theorem, [19]). Assume that condition (6.2) holds. If $E_{n}$ is a solution of (6.1), then either $E_{n}=0$ for all $n \rightarrow \infty$, or

$$
\lim _{n \rightarrow \infty} \sqrt[n]{\left\|E_{n}\right\|}
$$

or

$$
\lim _{n \rightarrow \infty} \frac{\left\|E_{n+1}\right\|}{\left\|E_{n}\right\|}
$$

exists and is equal to modulus of one of the eigenvalues of matrix A.

Theorem 6.2. Suppose that $0<p<\frac{1}{2}$ and $\left\{\left(t_{n}, z_{n}\right)\right\}$ be a solution of the system (2.1) such that $\lim _{n \rightarrow \infty} t_{n}=\bar{t}$ and $\lim _{n \rightarrow \infty} z_{n}=\bar{z}$. Then the error vector

$$
E_{n}=\left(\begin{array}{c}
e_{n}^{1} \\
e_{n-1}^{1} \\
e_{n}^{2} \\
e_{n-1}^{2}
\end{array}\right)=\left(\begin{array}{c}
t_{n}-\bar{t} \\
t_{n-1}-\bar{t} \\
z_{n}-\bar{z} \\
z_{n-1}-\bar{z}
\end{array}\right)
$$

of every solution of system (2.1) satisfies both of the following asymptotic relations:

$$
\begin{aligned}
& \lim _{n \rightarrow \infty} \sqrt[n]{\left\|E_{n}\right\|}=\left|\lambda_{1,2,3,4} F_{J}(\bar{t}, \bar{z})\right|, \\
& \lim _{n \rightarrow \infty} \frac{\left\|E_{n+1}\right\|}{\left\|E_{n}\right\|}=\left|\lambda_{1,2,3,4} F_{J}(\bar{t}, \bar{z})\right|,
\end{aligned}
$$

where $\lambda_{1,2,3,4} F_{J}(\bar{t}, \bar{z})$ are the characteristic roots of the Jacobian matrix $F_{J}(\bar{t}, \bar{z})$.

Proof. To find the error terms, we set

$$
\begin{aligned}
t_{n+1}-\bar{t} & =\sum_{i=0}^{1} \alpha_{i}\left(t_{n-i}-\bar{t}\right)+\sum_{i=0}^{1} \beta_{i}\left(z_{n-i}-\bar{z}\right), \\
z_{n+1}-\bar{z} & =\sum_{i=0}^{1} \gamma_{i}\left(t_{n-i}-\bar{t}\right)+\sum_{i=0}^{1} \delta_{i}\left(z_{n-i}-\bar{z}\right),
\end{aligned}
$$

and $e_{n}^{1}=t_{n}-\bar{t}, e_{n}^{2}=z_{n}-\bar{z}$. Thus we have

$$
\begin{aligned}
& e_{n+1}^{1}=\sum_{i=0}^{1} \alpha_{i} e_{n-i}^{1}+\sum_{i=0}^{1} \beta_{i} e_{n-i}^{2} \\
& e_{n+1}^{2}=\sum_{i=0}^{1} \gamma_{i} e_{n-i}^{1}+\sum_{i=0}^{1} \delta_{i} e_{n-i}^{2}
\end{aligned}
$$

where

$$
\begin{aligned}
\alpha_{0} & =\alpha_{1}=0, \\
\beta_{0} & =\frac{p}{z_{n-1}^{2}}, \beta_{1}=\frac{-p\left(\bar{z}+z_{n-1}\right)}{\bar{z} z_{n-1}^{2}}, \\
\gamma_{0} & =\frac{p}{t_{n-1}^{2}}, \gamma_{1}=\frac{-p\left(\bar{t}+t_{n-1}\right)}{\bar{t} t_{n-1}^{2}}, \\
\delta_{0} & =\delta_{1}=0 .
\end{aligned}
$$


Now we take the limits

$$
\begin{aligned}
\lim _{n \rightarrow \infty} \alpha_{0} & =\lim _{n \rightarrow \infty} \alpha_{1}=0, \\
\lim _{n \rightarrow \infty} \beta_{0} & =\frac{p}{\bar{z}^{2}}, \lim _{n \rightarrow \infty} \beta_{1}=\frac{-2 p}{\bar{z}^{2}}, \\
\lim _{n \rightarrow \infty} \gamma_{0} & =\frac{p}{\bar{t}^{2}}, \lim _{n \rightarrow \infty} \gamma_{1}=\frac{-2 p}{\bar{t}^{2}}, \\
\lim _{n \rightarrow \infty} \delta_{0} & =\lim _{n \rightarrow \infty} \delta_{1}=0 .
\end{aligned}
$$

Hence

$$
\begin{aligned}
& \beta_{0}=\frac{p}{\bar{z}^{2}}+a_{n}, \beta_{1}=\frac{-2 p}{\bar{z}^{2}}+b_{n}, \\
& \gamma_{0}=\frac{p}{\bar{t}^{2}}+c_{n}, \gamma_{1}=\frac{-2 p}{\bar{t}^{2}}+d_{n}
\end{aligned}
$$

where $a_{n} \rightarrow 0, b_{n} \rightarrow 0, c_{n} \rightarrow 0, d_{n} \rightarrow 0$ as $n \rightarrow \infty$. Therefore, we obtain the system of the form (6.1)

$$
E_{n+1}=(A+B(n)) E_{n}
$$

where

$$
\begin{aligned}
A & =\left(\begin{array}{cccc}
0 & 0 & \frac{p}{\bar{z}^{2}} & \frac{-2 p}{\bar{z}^{2}} \\
1 & 0 & 0 & 0 \\
\frac{p}{t^{2}} & \frac{-2 p}{t^{2}} & 0 & 0 \\
0 & 0 & 1 & 0
\end{array}\right), \\
B(n) & =\left(\begin{array}{cccc}
0 & 0 & a_{n} & b_{n} \\
1 & 0 & 0 & 0 \\
c_{n} & d_{n} & 0 & 0 \\
0 & 0 & 1 & 0
\end{array}\right),
\end{aligned}
$$

and $\|B(n)\| \rightarrow 0$ as $n \rightarrow \infty$. So, the limiting system of error terms about the equilibrium point $(\bar{t}, \bar{z})$ can be written as follows:

$$
\left(\begin{array}{c}
e_{n+1}^{1} \\
e_{n}^{1} \\
e_{n+1}^{2} \\
e_{n}^{2}
\end{array}\right)=\left(\begin{array}{cccc}
0 & 0 & \frac{p}{\bar{z}^{2}} & \frac{-2 p}{\bar{z}^{2}} \\
1 & 0 & 0 & 0 \\
\frac{p}{t^{2}} & \frac{-2 p}{t^{2}} & 0 & 0 \\
0 & 0 & 1 & 0
\end{array}\right)\left(\begin{array}{c}
e_{n}^{1} \\
e_{n-1}^{1} \\
e_{n}^{2} \\
e_{n-1}^{2}
\end{array}\right),
$$

which is same as linearized system of system (2.1) about equilibrium point $(\bar{t}, \bar{z})$.

\section{Numerical Simulations}

Example 7.1. We consider system (2.1) for $p=0.43$. With the initial values $t_{-1}=1, t_{0}=1.2, z_{-1}=3$ and $z_{0}=0.95$, positive equilibrium point of system (2.1) is globally asymptotically stable. Figure 1 and Figure 2 verify our theoretical results.

Example 7.2. We consider system (2.1) for $p=2.2$. With the initial values $t_{-1}=2.08, t_{0}=2.02, z_{-1}=2.03$ and $z_{0}=2.08$, solutions of system (2.1) oscillate about positive equilibrium point $(\bar{t}, \bar{z})=(2.0652,2.0652)$. Figure 3 verifies our theoretical results. 


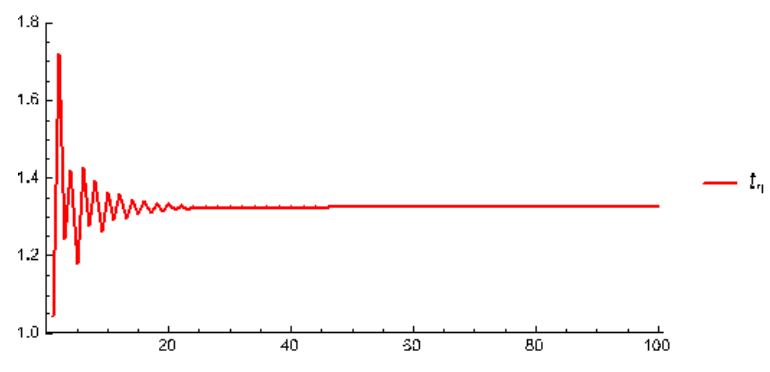

Figure 1. Plot of $t_{n}$.

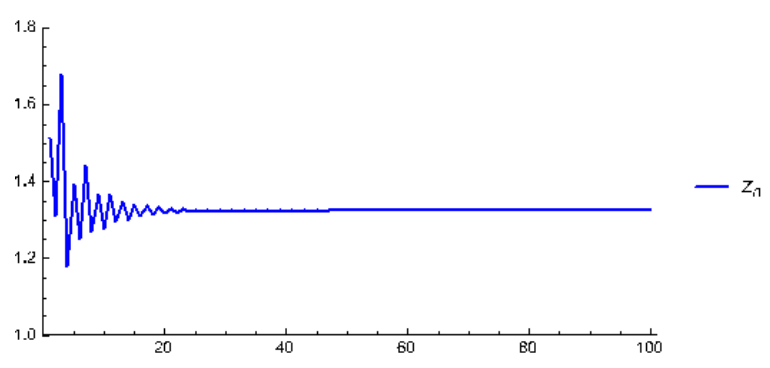

Figure 2. Plot of $z_{n}$.

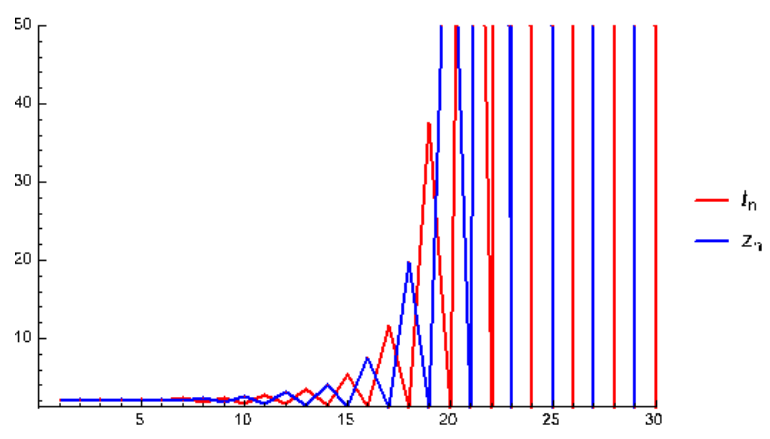

Figure 3. Plot of system (2.1)

\section{Conclusions}

This proposed study is related to convergence results of a system of second order difference equations. Firstly we investigate the unique positive equilibrium point of system (2.1). Then we analyse the bounded solutions of system (2.1). We also study the oscillation of solutions of system. Moreover we especially focus on the convergence results of solutions of system. According to our results, if $0<p<\frac{1}{2}$ then the positive equilibrium point of system (2.1) is globally asymptotically stable. After this we study the rate of convergence of solutions of system (2.1). In addition to this we present two numerical simulations to verify our theoretical results. 


\section{REFERENCES}

[1] Agarwal, R.P., Wong, P.J., Advanced topics in difference equations (Vol. 404), Springer Science \& Business Media, (2013).

[2] Bilgin, A, Kulenovic, M.R.S., Global Asymptotic Stability for Discrete Single Species Population Models, Discrete Dyn. Nat. Soc., (2017). https://doi.org/10.1155/2017/5963594

[3] Bacani, J. B., and J. F. T. Rabago. On two nonlinear difference equations. Dyn. Contin. Discrete Impuls. Syst. Ser. A Math. Anal., 24, 375-394, 2017.

[4] Bešo, E., Kalabušić, S., Mujić, N. Pilav E. Boundedness of solutions and stability of certain second-order difference equation with quadratic term. Adv. Differ. Equ. 2020, 19, 1-22 (2020). https://doi.org/10.1186/s13662-019-2490-9

[5] Burgić, Dž.; Kulenović, M. R. S.; Nurkanović, M. Global dynamics of a rational system of difference equations in the plane. Commun. Appl. Nonlinear Anal. 15(1), 71-84 (2008).

[6] Din, Q.: Asymptotic behavior of an anti-competitive system of second-order difference equations. J. Egyptian Math. Soc.. 24(1), 37-43 (2016).

[7] Din, Q., Elsayed, E.M.: Stability analysis of a discrete ecological model. Computational Ecology and Software. 4(2), 89-103 (2014).

[8] Elabbasy, E.M., Eleissawy, S.M. Asymptotic behavior of two dimensional rational system of difference equations. Dyn. Contin. Discrete Impuls. Syst. Ser. B Appl. Algorithms, 2013, 20: 221-235.

[9] Elaydi, S. An Introduction to Difference Equations, Springer-Verlag, New York, Inc, 1996.

[10] Garic-Demirovic, M., Hrustić, S, Morankic, S. (2019) Global dynamics of certain nonsymmetric second order difference equation with quadratic terms. Sarajevo J. Math., 15(2), $155-167$.

[11] Hadžiabdić, V., Kulenović, M.R.S., Pilav, E. Dynamics of a two-dimensional competitive system of rational difference equations with quadratic terms. Adv. Differ. Equ. 2014, 301, 1-32, (2014). https://doi.org/10.1186/1687-1847-2014-301

[12] Khan, A.Q., Qureshi, M.N.: Qualitative behavior of two systems of higher-order difference equations. Math. Methods Appl. Sci. 39(11), 3058-3074, (2016).

[13] Khan, AQ, Sharif, K. Global dynamics, forbidden set and transcritical bifurcation of a one-dimensional discrete-time laser model. Math. Meth. Appl. Sci. 2020; 1-13. https://doi.org/10.1002/mma.6201

[14] Kulenovic, M.R.S., Nurkanovic, M., Nurkanovic, Z. (2019) Global dynamics of certain mix monotone difference equation via center manifold theory and theory of monotone maps, Sarajevo J. Math., 15(2), 129-154.

[15] Kulenovic, M. R., \& Ladas, G. (2001). Dynamics of second order rational difference equations: with open problems and conjectures. Chapman and Hall/CRC.

[16] Kocic, V. L., and Gerasimos Ladas. Global behavior of nonlinear difference equations of higher order with applications. Vol. 256. Springer Science \& Business Media, 1993.

[17] Okumuş, İ., Soykan, Y.: Dynamical behavior of a system of three-dimensional nonlinear difference equations. Adv. Differ. Equ. 2018:224, 1-15 (2018).

[18] Papaschinopoulos G, Schinas CJ. On the system of two nonlinear difference equations $x_{n+1}=$ $A+x_{n-1} / y_{n}, y_{n+1}=A+y_{n-1} / x_{n}$. Int. J. Math. Math. Sci. 2000; 23(12):839-848.

[19] Pituk, M.: More on Poincare's and Perron's theorems for difference equations. J. Difference Equ. Appl. 8, 201-216 (2002).

[20] Qureshi, S.M., Khan, A.Q.: Global Dynamics of a 3x6 System of Difference Equations. Discrete Dyn. Nat. Soc. 2019, 1-14 (2019).

[21] Taşdemir, E.: On the Asymptotically Periodic Solutions of A Fifth Order Difference Equation. J. Math. Anal. 10(3), 100-111 (2019).

[22] Taşdemir, E.: Dynamics of a Second-Order System of Nonlinear Difference Equations. Preprints 2019, 2019100357 (doi: 10.20944/preprints201910.0357.v1).

[23] Taşdemir, E.: Stability and Periodic Nature of a System of Difference Equations. Preprints 2019, 2019100347 (doi: 10.20944/preprints201910.0347.v1).

[24] Yang, L., Yang, J., Dynamics of a system of two nonlinear difference equations. Int. J. Contemp. Math. Sciences, 2011, 6(5): 209-214.

[25] Zhang D, Ji W, Wang L, Li X. On the Symmetrical System of Rational Difference Equation $x_{n+1}=A+y_{n-k} / y_{n}, y_{n+1}=A+x_{n-k} / x_{n}$. Applied Mathematics. 2013; 4(5):834-837. 
[26] Zhang Y, Yang X, Evans DJ, Zhu C. On the nonlinear difference equation system $x_{n+1}=$ $A+x_{n-m} / y_{n}, y_{n+1}=A+y_{n-m} / x_{n}$. Comput. Math. Appl. 2007; 53:1561-1566.

Kirklareli University, Pinarhisar Vocational School, Kirklareli, 39300, Turkey

E-mail address, E. Taşdemir: erkantasdemir@hotmail.com 